\title{
Calibration system of EAS Cherenkov arrays using commercial drone helicopter
}

\author{
D. Voronin, ${ }^{a,}{ }^{*}$ A. Fazliakhmetov, ${ }^{a}$ N. Ushakov, ${ }^{a}$ A. Lukanov, ${ }^{a}$ B. Lubsandorzhiev, \\ $a$ R. Monkhoev $b$ and A. Sidorenkov $a$

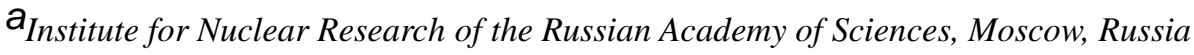

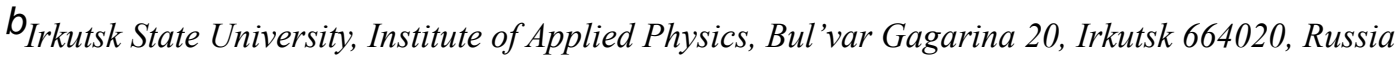 \\ E-mail: dmitry.m.voronin@gmail.com
}

EAS Cherenkov arrays are a powerful instrument for studies of primary cosmic rays in a wide range of energy. In this approach the Earth's atmosphere is used as a calorimeter providing EAS Cherenkov arrays high energy resolution. Another advantage of the method is its high time resolution which results in a good angular resolution. Usually EAS Cherenkov array is a sparsely instrumented array with a distance of $100 \mathrm{~m}$ (or more) between individual Cherenkov photon detectors (optical stations/modules) covering hundreds of square meters or a few thousands of square kilometers. So, to calibrate such arrays is not simple task. We developed a calibration system of EAS Cherenkov arrays based on a single fast light source on board of remotely controlled commercial drone helicopter. The light source is based on a single high power blue InGaN LED driven by avalanche transistors driver. The light source provides light pulses with 2-3 ns (FWHM) width and $10^{10}-10^{11}$ photons per pulse. Preliminary results of test flights of the calibration system are presented.

37th International Cosmic Ray Conference - ICRC2021

12-23 July, 2021

Berlin, Germany 


\section{Introduction}

Registration of Cherenkov light from extensive air showers (EAS) is a powerful tool for studies of high energy cosmic rays. Timing calibration in astroparticle physics experiments with sparsely distributed photon detectors like in EAS Cherenkov wide-angle arrays is not simple experimental task. Indeed spacing between photon detectors in EAS Cherenkov arrays operating in the energy range of cosmic rays $10^{15}-10^{17} \mathrm{eV}$ is in the order of $100 \mathrm{~m}$ or more, for higher energies the spacing is much more exceeding $1 \mathrm{~km}$. How to carry out timing calibration of individual photon detectors of such arrays, i.e. how to define simultaneousness of photon detectors firing or in other words how to define $T 0$ for all photon detectors of the array. The problem is far from trivial. In this case to use a single very powerful light source like powerful fast laser is problematic and expensive. The advent of ultra bright and high power fast blue/UV light emission diodes (LEDs) opened new possibilities for designing timing calibration systems for such kind of experiments [1-5].

\section{High power blue LEDs and their drivers}

Relatively new high power blue LEDs open new possibilities for design of Cherenkov detectors calibration systems. They are very bright and can withstand up to 1 A DC current and even higher. For EAS Cherenkov wide-angle arrays we have developed powerful nanosecond light source based on high power LEDs, for example LED XR7090 produced by Cree Company. The maximum of light emission spectrum of the LED is reached at $450 \mathrm{~nm}$, so called "Royal Blue" LED. To get high light yield of the light source staying still in a few nanoseconds time domain the LED is driven by specially designed driver (Fig. 1) using a pair of avalanche transistors ZTX415(FMMT415) switched consecutively. A positive triggering pulse causes consecutive avalanche breakdowns of the transistors which discharge $\mathrm{C} 2$ capacitor $(\mathrm{C} 2=47 \mathrm{pF})$ through high power LED providing nanosecond pulses of high light yield. The driver is triggered by a positive pulse with amplitude of $\geq 3 \mathrm{~V}$. The light source is stable over wide range of repetition rate up to $1 \mathrm{MHz}$, although usually in the calibration system of such kind of experiments the rate is quite low $\sim 5 \mathrm{~Hz}$.

The light yield of the light source measured by an integrating sphere is $\sim 10^{12}$ photons per pulse [3].

The light emission kinetics of the light source was measured by time correlated single photon counting technique (TCSPCT) and shown in Fig. 2.

Fast and slow components of the light emission kinetics are clearly seen. The substantial contribution to the total light yield belongs to the fast component. The width of light pulses of the source is $\sim 2.5-3 \mathrm{~ns}$ (FWHM).

The light source emits photons into $120^{\circ}$ full angle. The LED driver enclosed into a metallic box with $40 \times 40 \times 25 \mathrm{~mm}^{3}$ sizes as it is demonstrated in Fig. 3. On the right from the light source the XR7090 high power LED is shown. The total weight of the light source in this case is less than $100 \mathrm{~g}$.

Two approaches have been conceived to make time synchronization wide-angle Cherenkov array's optical detectors using above described light source. 


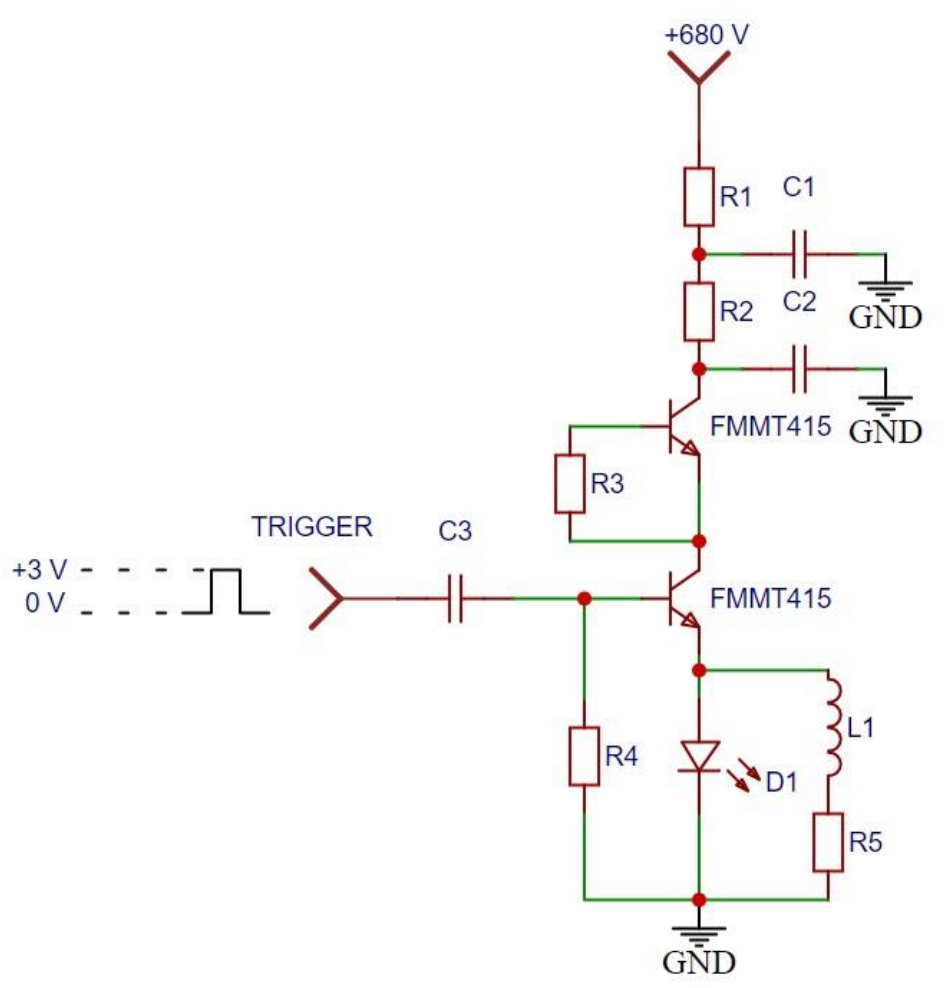

Figure 1: Electrical scheme of the LED driver for high power blue LED.

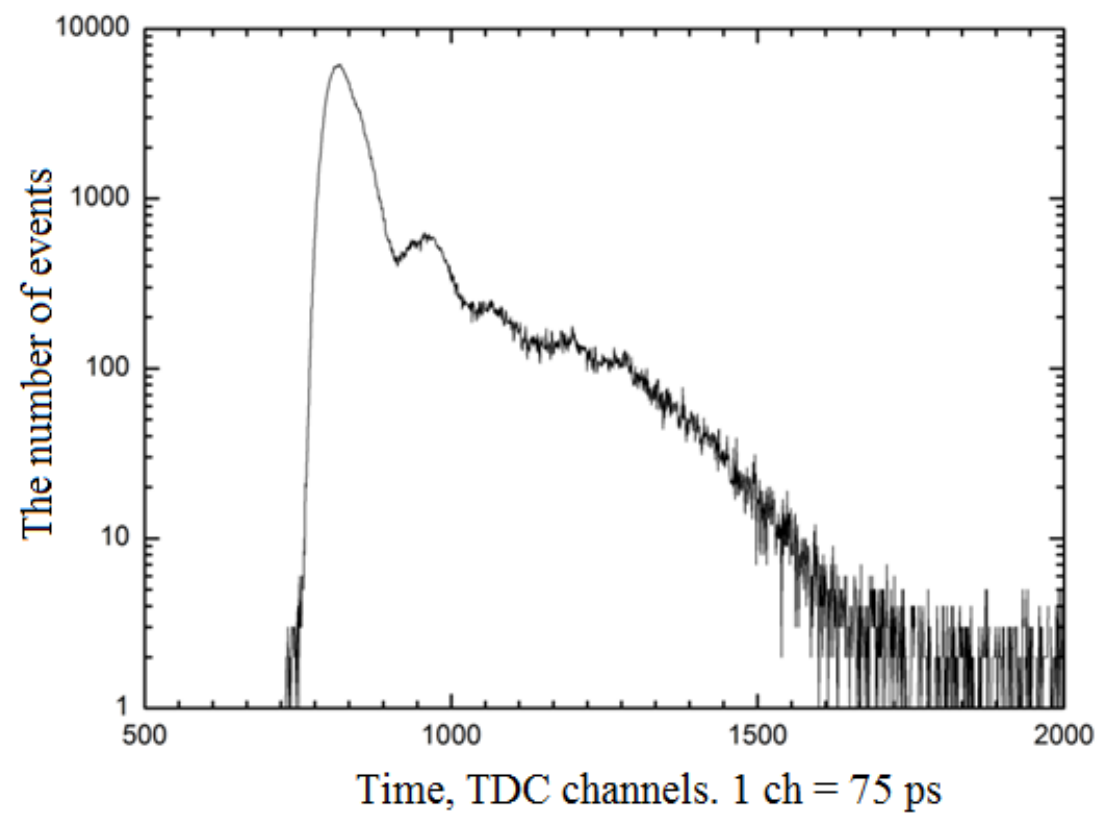

Figure 2: Light emission kinetics of high power LED driven by avalanche transistor driver. 


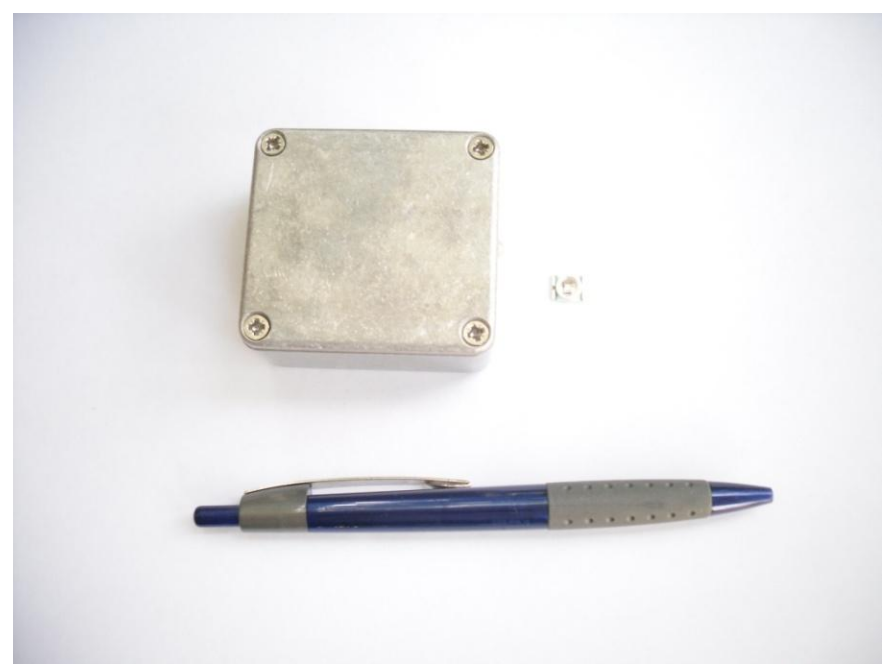

Figure 3: Calibration light source based on high power LED with avalanche transistor driver encapsulated into a metallic box. On the right high power LED itself.

The concept of the first approach was proposed more than 20 years ago by one of us (BL) and can be seen in Fig. 4. In this approach the light source is fixed on a helium balloon or a pilotless helicopter and raised at the height of $\sim 400 \mathrm{~m}$ above the array.

In this case one need to use GPS units and XBee radio units to know with good accuracy coordinates of the light source. In this approach it is enough to use only one light source because the light yield of the source and its emission angular distribution allow to illuminate from $400 \mathrm{~m}$ height all optical detectors of the array.

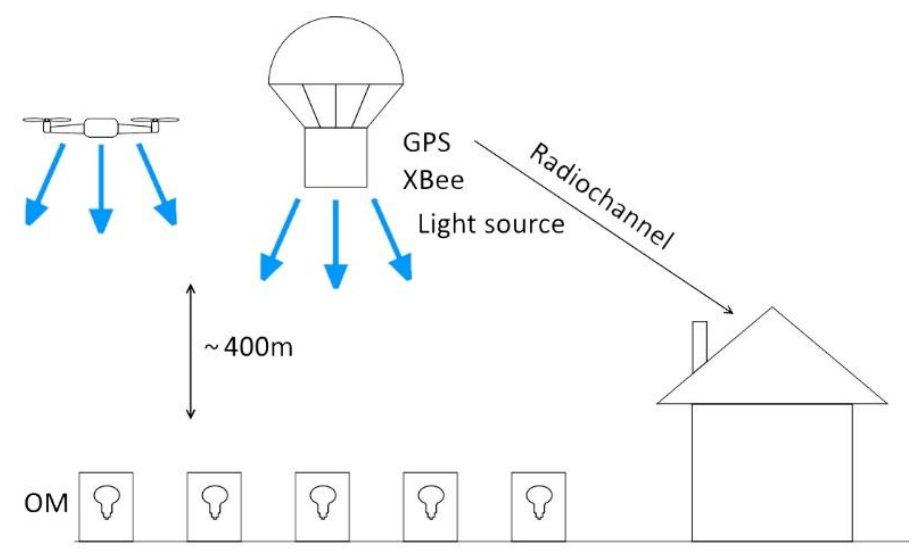

Figure 4: Calibration system concept based on light source elevated on balloon or dronehelicopter.

Implementation of the approach based on a balloon is hindered by the fact that so far it's unclear with which precision the coordinates of the light source on the balloon can be maintained. Another shortcoming of the approach is its price.

So, using a drone helicopter looks very promising. Indeed, they are easily available and relatively cheap even high quality professional ones. Even with a toy drone helicopter equipped one with a professional GNSS receiver can reach high precision of its location using RTK (Real 
Time Kinematics) technique. With this technique it is possible to reach a few $\mathrm{cm}$ precision of the drone location. One of drone helicopters which we are going to use for calibration of EAS Cherenkov arrays is shown in Fig. 5.

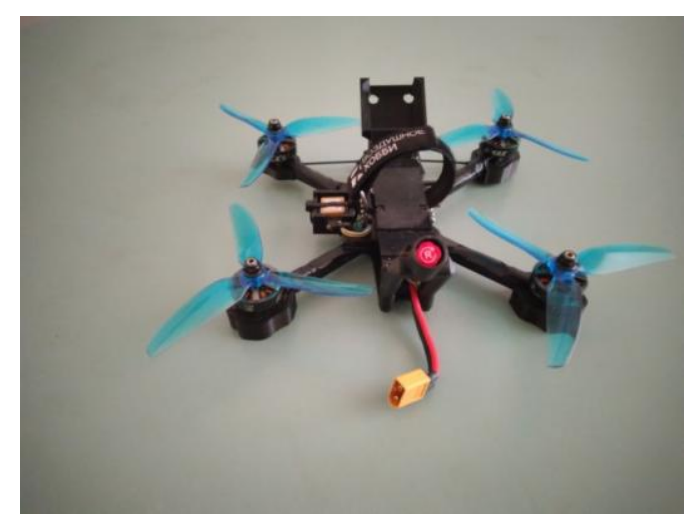

Figure 5: Toy drone helicopter.

The drone can reach easily $500 \mathrm{~m}$ hight above EAS Cherenkov array and elevate up to 300 $\mathrm{g}$ loading weight. The light source with its power supply and triggering pulse generator weighs well below $100 \mathrm{~g}$. The pulse generator is based on plate with 32-bit microcontroller STM32F103 (STMicroelectronics).

The light source power supply is High Voltage A series (XP Power). Its output voltage can be adjusted in the range of $0-800 \mathrm{~V}$. The out voltage polarity is positive. The weight of the power supply is less than 6 g. Fig. 6 shows a project of a PCB $\left(57 \times 43 \mathrm{~mm}^{2}\right)$ with an installed converter, LED driver, memory card slot, and a GNSS receiver connection is also provided. The drone location data will be recorded on the memory card for further analysis of the calibration data of EAS Cherenkov arrays.

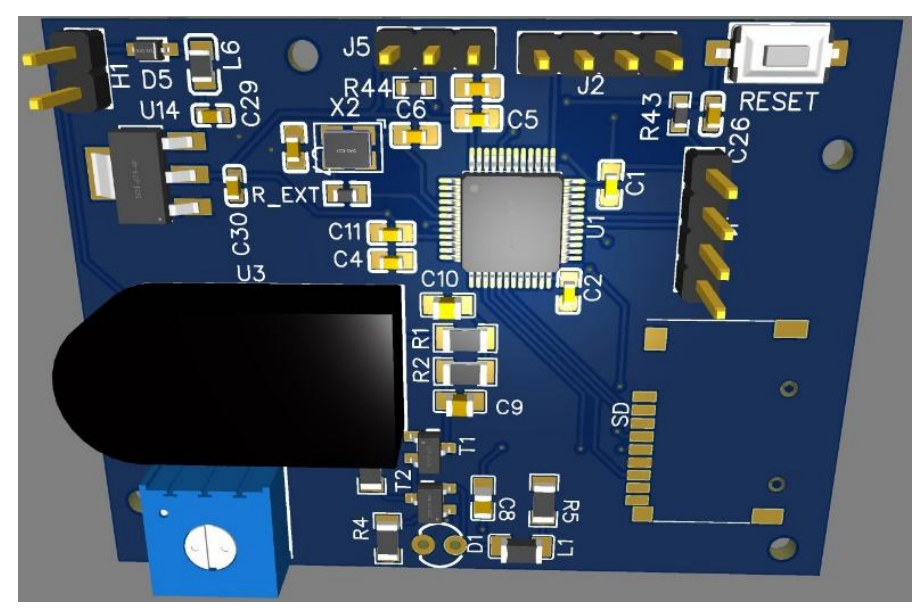

Figure 6: PCB for calibration. 


\section{Conclusion}

We developed a system based fast high power LED light source on board of a drone helicopter. The system performance is adequate for the requirements of EAS Cherenkov arrays.

\section{Acknowledgments}

This work was supported by the Russian Foundation for Basic Research (grant \#20-3290179) and the Russian Federation Ministry of Science and High Education (FZZE-2020-0024).

\section{References}

[1] R. Vasilyev, B. Lubsandorzhiev and G. Pokhil, A nanosecond light source for scintillation-and Cerenkov-detector calibration, Instruments and Experimental Techniques 43 (2000) 570.

[2] E. Vyatchin and B. Lubsandorzhiev, Studying the stability of nanosecond light-pulse sources based on blue GaN and InGaN light-emitting diodes, Instruments and Experimental Techniques 47 (2004) 493.

[3] B. Lubsandorzhiev, R. Poleshuk, B. Shaibonov and Y. Vyatchin, LED based powerful nanosecond light sources for calibration systems of deep underwater neutrino telescopes, Nuclear Instruments \& Methods in Physics Research Section A-accelerators Spectrometers Detectors and Associated Equipment 602 (2009) 220.

[4] Y.E. Vyatchin. PhD Thesis, Moscow, 2006. In Russian.

[5] B.A. Shaibonov, PhD Thesis, Moscow, 2010. In Russian. 